\title{
Extending the Use of HTS to Feeders in Superconducting Magnet Systems
}

\author{
Amalia Ballarino, Karl Hubert Meß, and Thomas Taylor
}

\begin{abstract}
Following the successful adoption of high temperature superconductors (HTS) in over a thousand current leads that will feed 3 MA from warm to cold in the Large Hadron Collider (LHC), the use of HTS has been generally accepted as suitable technology for the design of efficient leads feeding cryo-magnets. We now consider the extension of the technology to the interconnection of strings of superconducting magnets and their connection to feed-boxes through which the excitation current is fed. It is proposed to use HTS material for this application instead of low-temperature superconductor or normal-conducting material. The implications of adopting this technology are discussed with regard to the choice of materials, highlighting the differences with more conventional schemes. Examples are given of how this approach could be applied to the consolidation and upgrade of the LHC.
\end{abstract}

Index Terms-DC powering, high temperature superconductors, particle accelerators, superconducting feeders.

\section{INTRODUCTION}

M ORE than a thousand High Temperature Superconducting (HTS) current leads, with a total capacity of over $3 \mathrm{MA}$, were installed in the LHC to conduct the current from room temperature down to the liquid helium environment of the LHC superconducting magnets [1], and the commissioning has started. While the use of HTS is now mature technology for leads feeding cryo-magnets, the electrical connection of strings of superconducting magnets and their connection to the current leads are at present accomplished with $\mathrm{Nb}$-Ti superconducting wire or cable, stabilized with copper or aluminum. It is essential to keep these conductors below about $6 \mathrm{~K}$, which is achieved efficiently by operating them in a bath of either liquid or supercritical helium. Indirect cooling by heat conduction to pipes filled with cryogens is more critical, with the limited temperature margin often being problematic. It can be difficult to guarantee that interconnects are sufficiently cold, in particular for conduction-cooled magnets, based on the use of cryo-coolers, and for gas cooled cables. It is interesting to consider the use of HTS material instead of Nb-Ti. This approach has some major implications, both concerning the choice of materials and the optimization of a design that takes into account the material constraints. Examples are given of how this technology could be usefully applied to systems being studied for the consolidation and upgrade of the LHC.

Manuscript received August 27, 2007.

The authors are with CERN, the European Organization for Nuclear Research, 1211 Geneva 23, Switzerland (e-mail: amalia.ballarino@cern.ch; karl. hubert.mess@cern.ch; tom.taylor@cern.ch).

Color versions of one or more of the figures in this paper are available online at http://ieeexplore.ieee.org.

Digital Object Identifier 10.1109/TASC.2008.920622

\section{APPLICATION OF HTS CABLES to ACCELERATORS}

\section{A. Perceived Requirements}

It is first instructive to survey where HTS technology would be useful to resolve impending problems, and where it could change the approach to designing the system.

1) The powering of the magnets is presently done via current leads housed in cryostats in line with the beam [2]. Increasing beam intensities will result in an increase in dose of ionizing radiation. Besides the direct heat load and the impact on the choice of materials, parts of the feedboxes will be activated and consequently difficult to maintain. This is a strong motivation to remove "active" parts of the electrical feedboxes away from the beam, to places where they can be shielded.

2) HTS leads have lower heat losses than conventional leads [3]. Higher currents can therefore be envisaged in the magnets, reducing the inductance and thus easing the problem of quench protection. The temperature constraints at the lower end of the leads could be relaxed by introducing HTS in replacement of the Low Temperature Superconducting (LTS) bus.

3) LTS bus work associated with conduction-cooled magnets requires a sophisticated cooling approach to avoid quenches. This is rendered less critical if HTS material is used.

4) HTS cables will be required for connecting strings of superconducting magnets made with HTS coils.

5) Small high field magnets that are required in places with limited access could also be made using coils of HTS material. This has the advantage that the cryostat could be simplified, and the coils cooled by conduction (possibly using a cryo-cooler). It would need a superconducting bus.

6) Corrector magnets, operating in a high background field and possibly exposed to a high particle flux, could be made with HTS material, also requiring an insensitive connection.

\section{B. Applications of HTS Cables to the LHC}

For the LHC, the following applications are identified:

1) To provide more flexibility in the location of the cryostats supporting the current leads (electrical feedboxes), in cases where space is limited and the radiation environment harsh, such as the vicinities of the interaction points and the beam collimation insertions.

2) To provide relatively long distance links between power converters and superconducting magnet systems, in replacement of warm bus-bars/cables or LTS cables. 
3) To link cold magnets electrically. In particular, this technology could be used to replace the "connection cryostats" (cryostats containing cold connections of several meters).

These are applications that could be of specific benefit to consolidation and upgrade work foreseen for the LHC. Conventional feedbox cryostats can be displaced within the accelerator tunnel, or removed from it to be placed in existing alcoves, parallel tunnels or underground halls. The resistive part of the leads, joined at their cold ends to the HTS bus-bars, can be installed in a relatively radiation-free, more accessible location, and optimized for operating with the cold end at a convenient temperature [3]. The advantages include removing sensitive elements (valves, electronics, heating control, etc.) away from the hostile environment close to the beam, reducing the volume and complexity of the equipment in the tunnel, and, with the removal of the bulky room temperature power cables from the proximity of the magnets, an important saving of space. If the feedboxes are removed from the accelerator tunnel, a further advantage for machine operation is that the active components can be easily accessed at any time, even during runs with beam. In addition, the gain in temperature margin, as compared to the use of LTS cable, would make it possible to absorb additional transient heat loads (due to radiation losses, for example), and lead to more relaxed requirements for the cryogenic system.

\section{The Need for R\&D Targeted on This Requirement}

Considerable R\&D is being done on HTS cables for electrical utilities, and it might be thought that one could simply apply these technologies. However, at present this work is focused on replacing parts in existing sections of the grid. The typical applications are single or 3-phase AC conductors with high voltage insulation and liquid nitrogen cooling [4], [5]. Other systems, especially in association with particle accelerators, require the focus to be shifted to high quasi-DC current carrying capability, sometimes many cables in parallel, preferably cooled with liquid or gaseous helium.

In the CERN LHC, for example, there are many such connecting cables - over 50000 segments in fact, with a total length of $1360 \mathrm{~km}$, either made as flat-cables with 10 individual conductors, or as round cables with up to 48 insulated conductors. Thus, we seek to provide multiple circuits, electrically isolated at around $1 \mathrm{kV}$, carrying quasi-DC current: this requires a new approach to the design.

It should also be noted that the present approach by the electrical utilities to concentrate on HTS cables for transmission of AC current is sensible only for the replacement of relatively short lengths of existing parts of the grid. For large-scale application of HTS technology it would clearly be more appropriate to transmit heavy DC currents, placing AC-DC converters at the ends. $R \& D$ done in this domain for accelerators and other scientific applications therefore provides the potential for significant technology transfer to more classical electrical engineering applications.

\section{Materials, Design AND QUENCh PRotection}

\section{A. HTS Materials}

Four families of HTS materials can be envisaged as candidates for cable applications: Bi-2223 in Ag matrix, Bi-2212

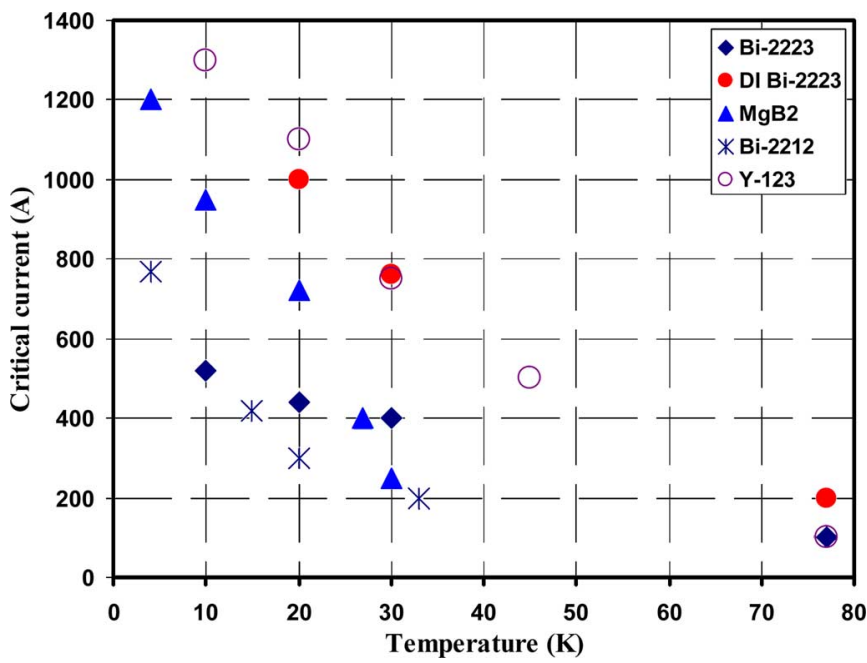

Fig. 1. Critical current of HTS materials as a function of temperature (self-field conditions).

in $\mathrm{Ag}$ matrix, 2nd generation $\mathrm{Y}-123$, and $\mathrm{MgB}_{2}$. The graph in Fig. 1 shows the critical current $\left(I_{c}\right)$ in self-field for engineering quality materials presently available in long lengths from Columbus $\left(\mathrm{MgB}_{2}\right)$, Sumitomo (DI Bi-2223), EHTS (Bi-2223), OST (Bi-2212), Superpower and AMSC (Y-123) [6]-[9].

In addition to providing liquid and superfluid helium, the LHC cryogenic system provides gaseous helium at 4.5 to $20 \mathrm{~K}$, used for the cooling of the beam screen and of the resistive part of the HTS leads, and at about $50 \mathrm{~K}$ for the cooling of the thermal screen of the cryostats. This provides very versatile cooling possibilities for the effective use of HTS materials - an attribute that was instrumental in the adoption of HTS technology for the current lead project. The operating temperature range for the $\mathrm{MgB}_{2}$ conductors also coincides with cooling using liquid hydrogen, with implications for eventual technology transfer.

For the application under discussion, it is foreseen to use gaseous helium in the lower temperature range. As can be seen in Fig. 1, operation in self or low field $(<100 \mathrm{mT})$, and with low temperature cooling (up to $\sim 20 \mathrm{~K}$ ), provides ideal boundary conditions for taking advantage of the electrical properties of HTS materials.

For the present application, the possibility is investigated of feeding 600 A circuits via an HTS conductor. In this view $\mathrm{MgB}_{2}$ is attractive, provided the maximum temperature is maintained below about $20 \mathrm{~K}$. Y-123 and Bi-2223 are also good candidates.

The most mature HTS material is Bi-2223, but the potential for cost reduction is limited due to its requiring a silver matrix. While potentially cheaper, Y-123 is proving difficult to produce. The more recently discovered $\mathrm{MgB}_{2}$ is both fairly easy to make and potentially inexpensive, but there is as yet little accumulated experience with its use. The choice of the material for the LHC application in study could fall on any one of these three materials, and will depend on the adaptability to this particular use in terms of feasibility for integration, quench protection and, finally, cost. 


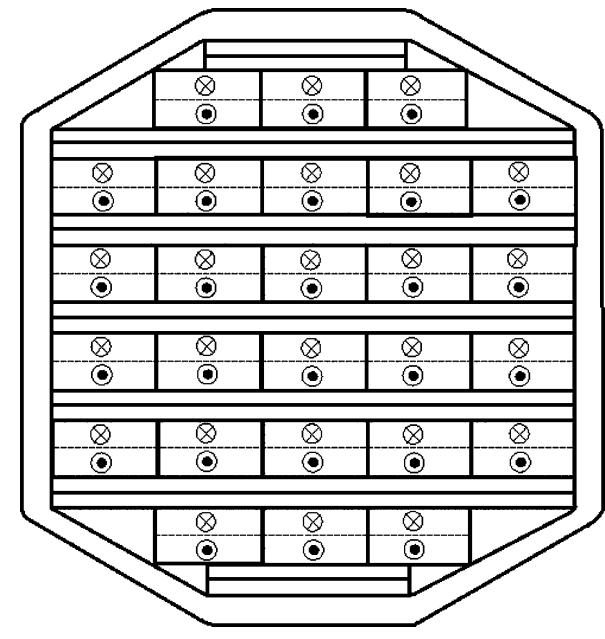

Fig. 2. Cross section of HTS cable with 26 pairs of 600 A conductors.

\section{B. Design}

The present medium term aim of the development is to develop a model multi-conductor cable that would test concepts being considered for the LHC (i) for the connection of a displaced electrical feedbox for the large aperture inner triplet system being studied for a luminosity upgrade, and (ii) to replace an existing $\mathrm{Nb}-$ Ti-based current link [2] that is located in the vicinity of collimators, and which risks being subjected to bursts of intense radiation heating. In the first case two 13000 A conductors together with several rated at $600 \mathrm{~A}$ will be required, and in the second case 48 of capacity $600 \mathrm{~A}$. The design is such that a similar cable could possibly be configured to serve both applications.

A sketch of the cross-section of a cable containing 26 pairs of 600 A conductors is shown in Fig. 2. Each conductor comprises 2 HTS tapes, together with copper tape for stabilization, insulated with a triple layer of polyimide tape. Conductors are paired to give a $600 \mathrm{~A}$ circuit that is also wrapped with two layers of polyimide tape, and the circuits are arranged with structural material in an array to form a rounded rectangular cable that is enclosed in a braided wrap for protection and handling. The cable sketched Fig. 2, which was designed to use Y-123 or Bi-2223 tapes with a cross section of $4 \mathrm{~mm} \times 0.2 \mathrm{~mm}$, has external dimensions of $25 \mathrm{~mm} \times 25 \mathrm{~mm}$. The cable also incorporates a superconducting quench prevention element that signals excessive temperature of the cryogen so that the current can be reduced before the critical current is attained, as well as signal wires connected to the far end of the conductors for quench detection. Differential thermal contraction of the cable with respect to the room temperature environment is handled by providing appropriate space within the enclosing pipe through which the helium cooling gas circulates. In straight runs, snaking with a period of $1 \mathrm{~m}$ within a pipe of $70 \mathrm{~mm}$ diameter will create sufficient slack; in runs of multiple curves, the slack can be provided by placing the warm bus off-centre in the pipe. For the straight runs the helium enclosure could be a smooth stainless steel pipe with periodic compensating bellows, continuous welded invar, or corrugated stainless steel. For runs consisting of a sequence

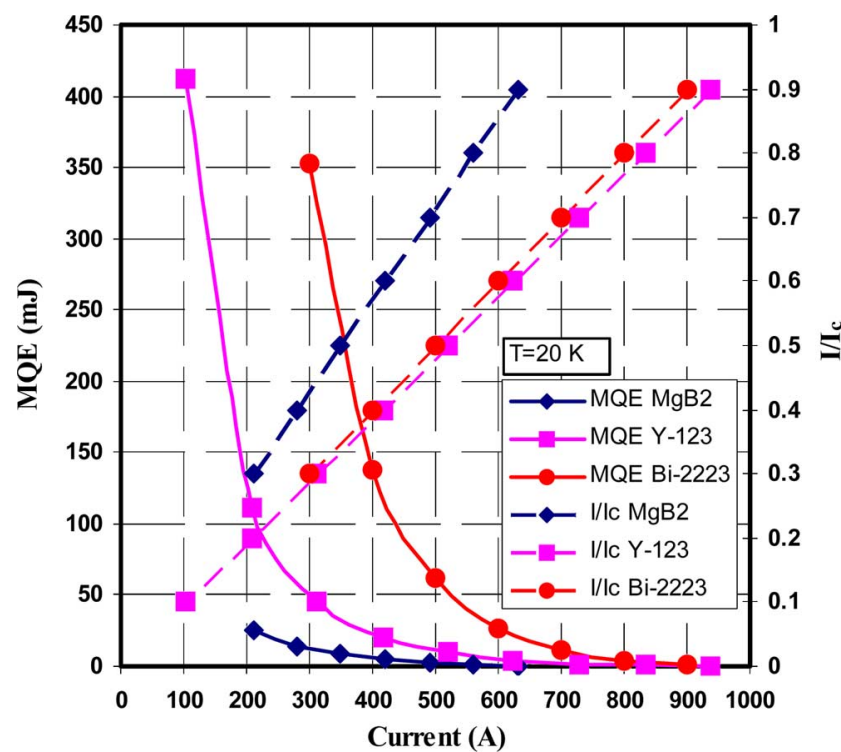

Fig. 3. Minimum quench energy (MQE) calculated at $20 \mathrm{~K}$ and normalized current $\left(\mathrm{I} / \mathrm{I}_{\mathrm{c}}\right)$ of MgB2, Y-123 and Bi-2223 tape as a function of operating current.

of curves (such as could be the case for connecting a remote feedbox) the corrugated stainless steel solution would be clearly advantageous.

\section{Stability and Quench Protection}

The quench behavior of an extended HTS object is determined by its critical current and temperature, its n-value and its operating current and temperature, as well as by the cross section, and the thermal and electrical properties of the constituent materials.

A parameter used for comparing stability of conductors is the minimum quench energy (MQE). Fig. 3 reports the MQE calculated for different conductors by using the material $\mathrm{I}_{\mathrm{C}}-\mathrm{T}$ values reported in Fig. 1, and the electrical and thermal properties measured on short samples over the range from $4.2 \mathrm{~K}$ to room temperature [10]. Although $\mathrm{MgB}_{2}$ in the operating range of interest for this application has a relatively low MQE (about $14 \mathrm{~mJ}$ at $300 \mathrm{~A}$ and $20 \mathrm{~K}$ ), it is nevertheless better than LTS [11]. Both Y-123 and Bi-2223 have higher MQE at the same operating current and temperature-about $50 \mathrm{~mJ}$ and $350 \mathrm{~mJ}$ respectively. The higher critical temperature of the HTS materials allows operation in the 10 to $20 \mathrm{~K}$ region, or above, where the specific heat of metals is already important, so that eventual deposited energy should be easily absorbed and conducted away. In the case of $\mathrm{MgB}_{2}$, the measured thermal conductivity has been found to be much lower than that calculated from its composition, possibly due to the unknown purity of metals and interaction at the interface layers in the matrix.

The cable is designed to ensure that the likelihood of a quench is low, but should a transition above the critical temperature occur, enough stabilizer is incorporated to limit the hot spot to below room temperature. For instance, for the LHC $600 \mathrm{~A}$ circuits in study, the longest discharge time constant of the circuits, which contain energy extraction, is $1.3 \mathrm{~s}$. The $\mathrm{MgB}_{2}$ tape of cross section of about $3.5 \mathrm{~mm} \times 0.65 \mathrm{~mm}$ contains enough 
stabilizer to satisfy this constraint, while for the Bi-2223 and Y-123 conductors it is necessary to add some thin copper tapes.

Quench detection will be based on the detection of the resistive voltage across the cable. Typically an instrumentation amplifier will be used, followed by an Analogue to Digital Converter and eventually a digital signal processor. The processor gives the possibility to improve the signal-to-noise ratio, if needed. The routing of the sensing cables is, however, most important. The inductance of the measurement setup, including the superconducting part, must be as small as possible to minimize the noise. For this reason the sense wires will be located within the bus-bar cable.

The low n-value of the Y-123 and Bi-2223 (20-30) enables the protection of the HTS device at a low voltage signal integrated in time in the current sharing regime of the superconductor. For this reason, the HTS part of the LHC leads, based on $\mathrm{Bi}-2223 \mathrm{Ag}-\mathrm{Au}$ tapes, is protected at $3 \mathrm{mV}$ threshold with a detection time of $50 \mathrm{~ms}$. The noise at the input, which could be problematic for such a low voltage, has been measured in the LHC commissioning phase during operation of the leads in the tunnel, and was found to be well below $100 \mu \mathrm{V}$. For these low n-value cables, it is advantageous to increase the amount of superconductor to maximize the likelihood of having stable recovery conditions. For the $\mathrm{MgB}_{2}$, where n-values above 50 are expected, protection resembles more that of LTS, and the stabilizer serves solely to preserve the conductor against overheating following a quench. For the 600 A circuits of this study, a detection time of $50 \mathrm{~ms}$ can be applied, with a corresponding detection threshold at maximum current of about $30 \mathrm{mV}$ and a peak of temperature, reached during the current discharge, of $270 \mathrm{~K}$. Such detection time and threshold are not problematic for the electronics developed and used for the quench protection of the LHC superconducting magnets [12].

The HTS cable will have a dedicated quench protection system. On the basis of experience gained with the LHC leads, together with that made on similar equipment installed at Fermilab [13], it is planned to adopt a threshold in the range from 1 to $3 \mathrm{mV}$ for Bi-2223 and Y-123 conductors. For the $\mathrm{MgB}_{2}$ detection voltages of 20 to $30 \mathrm{mV}$ can be safely applied.

\section{FUTURE Plans}

In order to qualify conductor architecture and important engineering aspects of the design, it is proposed to assemble and test three 8-circuit (16 conductors) cables of significant length ( $\sim 5 \mathrm{~m}$ ) using Bi-2223 and Y-123 (for testing at up to $35 \mathrm{~K}$ ), and $\mathrm{MgB}_{2}$ (for testing at 5-20 K). The cables are based on the use of standard $4 \mathrm{~mm}$ wide tape conductor; the length of the test cables is chosen to verify that differential thermal contraction can be controlled reliably, taking into account the mechanical properties of materials, and to demonstrate a method for making the electrical joints at the two ends of the cables. As it is possible to make $\mathrm{MgB}_{2}$ in the form of round wire, as is already the case for $\mathrm{Bi}-2212$, we also plan to investigate a cable layout using such strand of these materials.

\section{CONCLUSION}

The possible application of HTS to electrical feeder systems for superconducting magnets, beyond that of the current leads, has been addressed. In the case of the LHC, the utility of this approach has been identified for certain consolidation and upgrade work, and design concepts are proposed for the cables. It is being planned to construct and test significant demonstration lengths of conductors in order to qualify the concept and compare the performance, in typical working conditions, of cables using the different materials.

\section{REFERENCES}

[1] A. Ballarino, "HTS in the LHC \& in the LHC upgrades," in Proc. WAMDO Workshop, 2006, pp. 61-63 [Online]. Available: http://www. wamdo-2006.web.cern.ch/wamdo-2006

[2] T. Goiffon, J. Lyngaa, L. Metral, A. Perin, P. Trilhe, and R. van Weelderen, "Conceptual design of the Cryogenic Electrical Feedboxes and the LHC Superconducting Links," in Proc. ICEC 20, Beijing, China, 2004, pp. 1059-1064.

[3] A. Ballarino, "HTS Current leads: Performance overview in different operating modes," IEEE Trans. Appl. Supercond., vol. 17, no. 2, pp. 2282-2285, Jun. 2007.

[4] T. Masuda et al., "Design and experimental results for Albany HTS cable," IEEE Trans. Appl. Supercond., vol. 15, no. 2, pp. 1806-1809, Jun. 2005.

[5] J. A. Demko et al., "Triaxial HTS cable for the AEO Bixby project," IEEE Trans. Appl. Supercond., vol. 17, no. 2, pp. 2047-2050, Jun. 2007.

[6] G. Grasso, "Present state of long-lengths of $\mathrm{MgB}_{2}$ superconducting tapes for DC and AD applications," in Proc. WAMS Workshop, 2004 [Online]. Available: http://www.amt.web.cern.ch/amt/events/workshops/WAMS2004/proceedings

[7] T. Kato et al., "Development of drastically innovative BSCCO (DIBSCCO) wire," SEI Tech. Rev., no. 62, pp. 10-14, Jun. 2006.

[8] H. Krauth, "Development of LTS and HTS conductors for accelerator magnets at EAS," in Proc. WAMS Workshop, 2004 [Online]. Available: http://www.amt.web.cern.ch/amt/events/workshops/WAMS2004/proceedings

[9] X. Xiong et al., "High throughput processing of long-length IBAD $\mathrm{MgO}$ and epi-buffer templates at Superpower," IEEE Trans. Appl. Supercond., vol. 17, no. 2, pp. 3375-3378, Jun. 2007.

[10] S. A. March, A. Ballarino, C. Beduz, Y. Yang, and K. H. Meß, "Towards the design of power switches utilizing HTS material," presented at the EUCAS 2007 Conf., , unpublished.

[11] K. H. Meß, P. Schmüser, and S. Wolf, Superconducting Accelerator Magnets. Singapore: World Scientific, 1996.

[12] R. Denz, "Electronic systems for the protection of superconducting elements in the LHC," IEEE Trans. Appl. Supercond., vol. 16, no. 2, pp. 1725-1728, Jun. 2006.

[13] S. Feher et al., "HTS Power leads test results," in Proc. PAC 1999, New York, 1999. 